\title{
Three-Dimensional Temperature Distribution Produced by a Moving Laser Beam
}

\author{
R. Uyhan \\ Mathematics Department, University of Suleyman Demirel, 32260 Isparta, Turkey \\ Correspondence should be addressed to R. Uyhan; ramazanuyhan@sdu.edu.tr \\ Received 6 September 2013; Accepted 5 November 2013 \\ Academic Editor: M. Montaz Ali
}

Copyright (C) 2013 R. Uyhan. This is an open access article distributed under the Creative Commons Attribution License, which permits unrestricted use, distribution, and reproduction in any medium, provided the original work is properly cited.

An axisymmetric laser beam, moving with constant speed, heats a thin infrared absorbing layer sandwiched between two plastic sheets. We use a simplified theoretical model to study the three-dimensional unsteady temperature field produced by the moving laser beam.

\section{Introduction}

High quality hard copy of electronically stored images can be produced by thermal dye diffusion printing [1]. Such copies are of near photographic quality and have been used in such diverse areas as medical imaging and credit card personalisation. A theoretical model of the laser induced thermal dye-diffusion process for a stationary axisymmetric laser was given by [2], based upon the ICI Imagedata L2T2 system as described by [3].

In the L2T2 system a thin $2 \mu \mathrm{m}$ dye layer is sandwiched between a $6 \mu \mathrm{m}$ layer of PET (polyethylene-tetrathalate) and a relatively thick receiver sheet of thickness $40 \mu \mathrm{m}$. Heat is supplied directly to the dye-coat layer by a laser (see Figure 1). A suitable infrared absorbing material such as substituted copper phthalocyanine is incorporated into the dye coat layer which absorbs radiation strongly at about $800 \mathrm{~nm}$ [3]. The dye therefore diffuses rapidly into the receiver sheet producing a small dye dot. The total amount of dye transferred depends on the temperature because the diffusion coefficient is known experimentally to be a sensitive function of temperature [4]. In the L2T2 system there is a thin barrier layer at the PET/dye layer interface which prevents dye diffusing into the PET film.

The required image is formed by a large number of small dye dots produced in the receiver sheet as the pulsed laser beam moves relative to the layered system. In [2] a simplified model of the thermal dye diffusion process was studied in which the laser beam was fixed relative to the receiver sheet over the heating period typically $100 \mu$ s. The laser beam was then moved to the next pixel and the process was repeated and so on, producing a series of dots. It was assumed that there was no thermal interaction between adjacent pixels, so that we could consider the thermal dye diffusion process for a single typical pixel. In this case the time-dependent temperature and dye diffusion fields produced by the laser beam are axi-symmetric. A review of previous theoretical studies is given in $[2,5]$.

In practice the axisymmetric laser beam is moved continuously relative to the receiver sheet and is switched on and off in a periodic fashion, so that a series of dots is produced. In this situation both the temperature and the dye diffusion fields will depend on three space coordinates and time, so the task of finding the temperature and dye fields is more difficult. In this paper we will use a simple mathematical model to study the temperature field.

The temperature field produced by a Gaussian laser beam is of interest in other practical applications. Thus, for example, [6] studied the temperature field when the beam moves over the surface of a metal sheet of finite thickness, a study relevant to metal forming, while [7] studied the temperature field in a thin metal sheet, so that it could be assumed that the temperature was constant across it. In both these studies the beam had constant wattage and moved with constant speed, so that the temperature distribution took the form of a travelling wave. 


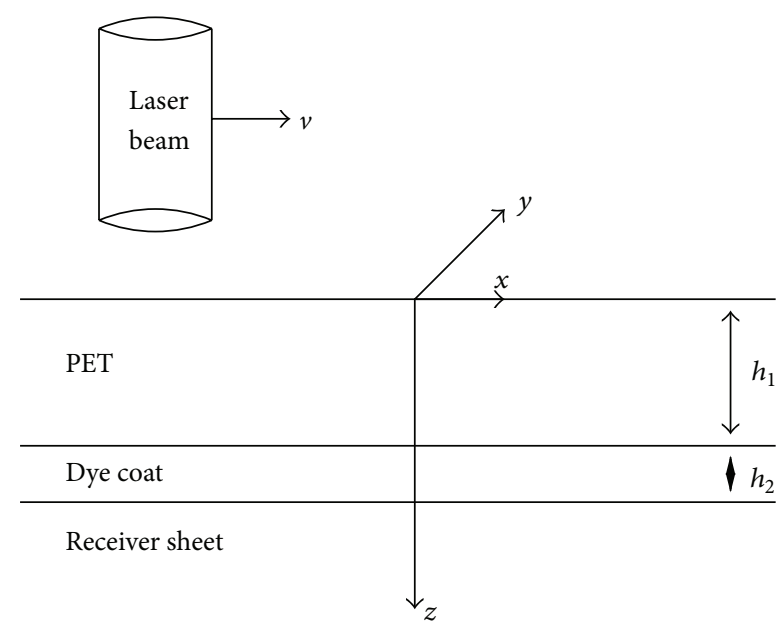

FIGURE 1: Illustration of the geometrical situation (not to scale).

In the present case the polymer layers have relatively low conductivity. As a consequence the receiver sheet of thickness $40 \mu \mathrm{m}$ can be regarded as infinitely thick for all practical purposes over the time scales of interest, but the finite thickness of the top $6 \mu \mathrm{m}$ PET layer, above the dye layer, has to be taken into account.

\section{The Mathematical Model}

Consider the layered system illustrated in Figure 1. The $z$ coordinate is measured into the layered system from the air interface which is situated at $z=0$. The top (PET) carrier layer has thickness $h_{1}$ and the dye-coat layer has thickness $h_{2}$. The receiver sheet is relatively thick (about $40 \mu \mathrm{m}$ ) and can be regarded as infinitely thick in practice. We take a frame of reference in which the polymer sheet is stationary and the laser beam is moved with constant speed $v$ in the positive $x$ direction over the surface $z=0$. We will assume that the axis of the laser is at $x=0, y=0$ at time $t=0$, so that at time $\tau$, its axis will be at $x=v \tau, y=0$. It is assumed in this paper that the thermal properties of the three layers shown in Figure 1 have the same constant values.

Consider first the absorption of laser radiation by the infrared absorber in the dye-coat layer. Assuming that the distribution of the absorber is uniform, then the laser radiation flux per unit area, $I(r, z, \tau)$ at time $\tau$, satisfies the differential equation:

$$
\frac{\partial I}{\partial z}=-\frac{\lambda}{h_{2}} I
$$

for $h_{1} \leq z \leq h_{1}+h_{2}$ where $\lambda$ is a constant. The quantity which is measured experimentally is the optical density which equals $\lambda / \ln 10$. Assuming that the radial dependence of the radiation flux on $z=h_{1}$ is Gaussian, it follows that, at time $\tau$,

$$
I(r, z, \tau)=I_{0} \exp \left(-\frac{\lambda\left(z-h_{1}\right)}{h_{2}}-\frac{r^{2}}{r_{0}^{2}}\right)
$$

where $I_{0}$ depends on $\tau, r_{0}$ is a characteristic radius for the moving beam, and $r^{2}=(x-v \tau)^{2}+y^{2}$. The wattage of the beam $W(\tau)$ is found by integrating $2 \pi r I(r, z, \tau)$ radially over the beam at $z=h_{1}$, and we find that $W=\pi r_{0}^{2} I_{0}$. It follows that the laser energy absorbed per unit volume per unit time in the dye layer is

$$
-\frac{\partial I}{\partial z}=\frac{W(\tau) \lambda}{\pi r_{0}^{2} h_{2}} \exp \left(-\frac{\lambda\left(z-h_{1}\right)}{h_{2}}-\frac{r^{2}}{r_{0}^{2}}\right) .
$$

This is of course the largest at the top of the dye layer, $z=h_{1}$. We note that the total energy absorbed per unit area per unit time is

$$
\frac{W}{\pi r_{0}^{2}}\left(1-e^{-\lambda}\right) \exp \left(\frac{-r^{2}}{r_{0}^{2}}\right)
$$

This is the largest when $\lambda=\infty$, in which case all the heat is generated in an infinitely thin layer at $z=h_{1}$.

Since, by assumption, the thermal properties have the same constant values in the three layers, we can construct the temperature field produced by the moving laser by combining instantaneous source solutions.

At time $\tau$ and at $z=z^{\prime}$, the laser beam generates a heat source distribution in the dye layer $q\left(\tau, z^{\prime}\right) \exp \left(-r^{2} / r_{0}^{2}\right) d z^{\prime} d \tau$ over a short time $d \tau$ in a thin layer of thickness $d z^{\prime}$.

Then from (3),

$$
q\left(\tau, z^{\prime}\right)=\frac{W(\tau) \lambda}{\pi r_{0}^{2} h_{2}} \exp \left[-\frac{\lambda\left(z^{\prime}-h_{1}\right)}{h_{2}}\right] .
$$

If the medium is infinite, has uniform properties, and has zero initial temperature, the temperature field for $t>\tau$ is given by

$$
\begin{aligned}
& G\left(x, y, z, t, z^{\prime}, \tau\right) \\
& \quad=\frac{q\left(\tau, z^{\prime}\right) \gamma(u)}{2 \rho c_{p} \sqrt{\pi \alpha u}} \exp \left[-\frac{\left(z-z^{\prime}\right)^{2}}{4 \alpha u}-\frac{\gamma(u) r^{2}}{r_{0}^{2}}\right],
\end{aligned}
$$

where $u=t-\tau, \gamma=r_{0}^{2} /\left(r_{0}^{2}+4 \alpha u\right), \alpha$ is the thermal diffusivity, $\rho$ is the density, and $c_{p}$ is the specific heat.

The solution (6) satisfies the heat conduction equation

$$
\frac{\partial G}{\partial t}=\alpha\left\{\frac{\partial^{2} G}{\partial z^{2}}+\frac{1}{r} \frac{\partial}{\partial r}\left(r \frac{\partial G}{\partial r}\right)\right\} .
$$

As $t \rightarrow \tau$, we can see that $G \rightarrow 0$, except near $z=z^{\prime}$, where it is large, and then (6) takes the approximate form

$$
G \simeq \frac{q\left(\tau, z^{\prime}\right)}{2 \rho c_{p} \sqrt{\pi \alpha u}} \exp \left[-\frac{\left(z-z^{\prime}\right)^{2}}{4 \alpha u}-\frac{r^{2}}{r_{0}^{2}}\right],
$$

which is the corresponding "one-dimensional" heat source solution of

$$
\frac{\partial G}{\partial t}=\alpha \frac{\partial^{2} G}{\partial z^{2}}
$$


(see, e.g., $[8,9])$. If instead heat is produced throughout the dye-coat layer $h_{1}<z<h_{1}+h_{2}$, the corresponding temperature field $T$ can be found by integrating the instantaneous heat source solution $G$ with respect to $z^{\prime}$ from $h_{1}$ to $h_{1}+h_{2}$ and with respect to $\tau$ from $-\infty$ to the current time $t$.

Thus we find that

$$
\begin{aligned}
& T(x, y, z, t) \\
& =\int_{-\infty}^{t} \int_{h_{1}}^{h_{1}+h_{2}} \frac{q\left(\tau, z^{\prime}\right) \gamma(u)}{2 \rho c_{p} \sqrt{\pi \alpha u}} \\
& \quad \times \exp \left[-\frac{\left(z-z^{\prime}\right)^{2}}{4 \alpha u}-\frac{\gamma(u) r^{2}}{r_{0}^{2}}\right] d z^{\prime} d \tau,
\end{aligned}
$$

where $q\left(\tau, z^{\prime}\right)$ is defined in (5). This is the temperature field produced by a moving laser in an infinite medium initially at zero temperature.

In the real problem, the initial temperature is $T_{A}$ and there is no heat flux at $z=0$, the air-polymer interface. To incorporate this boundary condition into the model, we introduce an appropriate image heat source distribution in $z<0$. Then by symmetry, there will be no heat flux across $z=0$. The effect of this is to give, after some manipulation,

$$
\begin{aligned}
T-T_{A}=\int_{-\infty}^{t} \int_{h_{1}}^{h_{1}+h_{2}} & \frac{q\left(\tau, z^{\prime}\right) \gamma(u)}{2 \rho c_{p} \sqrt{\pi \alpha u}} \\
& \times f\left(z, z^{\prime}, u\right) \exp -\frac{\gamma(u) r^{2}}{r_{0}^{2}} d z^{\prime} d \tau,
\end{aligned}
$$

where

$$
f\left(z, z^{\prime}, u\right)=\exp \left(\frac{-\left(z-z^{\prime}\right)^{2}}{4 \alpha u}\right)+\exp \left(\frac{-\left(z+z^{\prime}\right)^{2}}{4 \alpha u}\right) .
$$

In normal operation the laser beam is switched on and off in a periodic fashion. Assuming that the period is $\tau_{p}$ and the laser is switched on for a time $\tau_{\text {on }}$ and that when it is operating $W=W_{0}$, a constant, then we can take

$$
W(\tau)= \begin{cases}W_{0}, & a(n) \leq \tau<b(n), \\ 0, & \text { otherwise, }\end{cases}
$$

where $a(n)=n \tau_{p}, b(n)=\min \left(a(n)+\tau_{\text {on }}, t\right)$, and $n$ takes all integer values from $-\infty$ to $n=n^{*}$, where $a\left(n^{*}\right) \leq \tau<a\left(n^{*}+\right.$ 1). Thus

$$
\begin{aligned}
& T-T_{A} \\
& =Q_{0} \sum_{n=-\infty}^{n^{*}} \int_{a(n)}^{b(n)} \int_{h_{1}}^{h_{1}+h_{2}} \frac{\gamma(u)}{\sqrt{\pi \alpha \tau}} f\left(z, z^{\prime}, u\right) \\
& \quad \times \exp \left[-\frac{\lambda\left(z^{\prime}-h_{1}\right)}{h_{2}}-\frac{\gamma(u) r^{2}}{r_{0}^{2}}\right] d z^{\prime} d \tau,
\end{aligned}
$$

where $u=t-\tau, r^{2}=(x-v \tau)^{2}+y^{2}$, and $Q_{0}=W_{0} \lambda / 2 \pi r_{0}^{2} h_{2} \rho c_{p}$.
For the L2T2 system, typical values of physical parameters are $v=0.15 \mathrm{~m} / \mathrm{s}, \tau_{p}=150 \mu \mathrm{s}, \tau_{\text {on }}=100 \mu \mathrm{s}, \rho=1.3 \times$ $10^{3} \mathrm{~kg} / \mathrm{m}^{3}, c_{p}=1.9 \times 10^{3} \mathrm{~J} / \mathrm{kg} \cdot \mathrm{K}, \alpha=3.2 \times 10^{-8} \mathrm{~m}^{2} \mathrm{~s}^{-1}, h_{1}=$ $6 \mu \mathrm{m}, h_{2}=2 \mu \mathrm{m}, W_{0}=75 \mathrm{~mW}, r_{0}=15 \mu \mathrm{m}, \mu=0.7, \lambda=1$, and $T_{A}=20^{\circ} \mathrm{C}$.

\section{Results and Discussion}

The double integral (14) was evaluated using a standard NAG library routine. For the values of the parameters chosen, we found that the number of terms in the infinite series is small. This is because the axis of the beam passes over any given point in a time of order $2 r_{0} / v=200 \mu \mathrm{s}$, which is comparable to the heating time $\tau_{\text {on }}=100 \mu \mathrm{s}$. Thus only a few laser pulses can affect the temperature at a given point.

Consider the temperature field at $y=0$ near $x=0$. The axis of the laser passes over $x=0$ at time $\tau=0$. However, the temperature field near $x=0$ will depend on the precise times when the laser was switched on and off. In our calculations we have assumed that the laser is switched on at $\tau=0, \pm \tau_{p}$, $2 \pm \tau_{p} \ldots$. In particular this means that the laser was turned off for $-50 \mu \mathrm{s}<\tau<0$ if $\tau_{\text {on }}=100 \mu$ s, and consequently the maximum temperature in the dye coat layer cannot be at $x=0$. Numerical calculation shows that the maximum temperature in the dye coat layer is near $x=2.5 \mu \mathrm{m}$, and hence our numerical results are shown for this value of $x$. The position of maximum temperature will depend on the parameter values chosen.

The $z$-temperature profile at various times for $x=2.5 \mu \mathrm{m}$, $y=0$ is shown in Figure 2. It can be seen that the temperature rises between $-100 \mu \mathrm{s}$ and $-50 \mu \mathrm{s}$ but then falls until $t=0$ because the laser is turned off. The temperature then rises rapidly to about $900^{\circ} \mathrm{C}$ over the next $100 \mu$ s because the laser is switched on at $\tau=0$, and its axis is close to $x=2.5 \mu \mathrm{m}$. The laser is then turned off again and the temperature falls slowly to $700^{\circ} \mathrm{C}$ at $t=200 \mu \mathrm{s}$, as heat conducts upwards and downwards from the dye coat layer, with negligible heat input from the laser. This is shown in Figure 3.

The $z$-temperature profiles shown in Figures 2 and 3 are complicated mainly because they show that at least two laser pulses contribute significantly. These profiles are much more complicated than these for a stationary laser (see Figures 4 and 5) reproduced from our paper [2] for the same heating time. Note that the maximum temperature is about $1000^{\circ} \mathrm{C}$ in this case. This suggests that for these standard values, the process of dye diffusion is not well described by our stationary laser model. In general the maximum temperatures will be reduced as $v$ increases from zero and so will be dye diffusion, which is a sensitive function of temperature.

The effect of an increase in the speed of the laser to $0.5 \mathrm{~m} / \mathrm{s}$ is illustrated in Figures 6 and 7. As before, the laser is turned off at $\tau=-50 \mu \mathrm{s}$, but now the laser beam axis is at $v \tau=-25 \mu \mathrm{m}$. The heating before this time is therefore unimportant since $x=2.5 \mu \mathrm{m}$ is well outside the laser beam. Note that we have chosen to show the $z$-temperature profiles at $x=2.5 \mu \mathrm{m}$, as in the standard case. The maximum temperatures will be at a slightly larger value of $x$. 


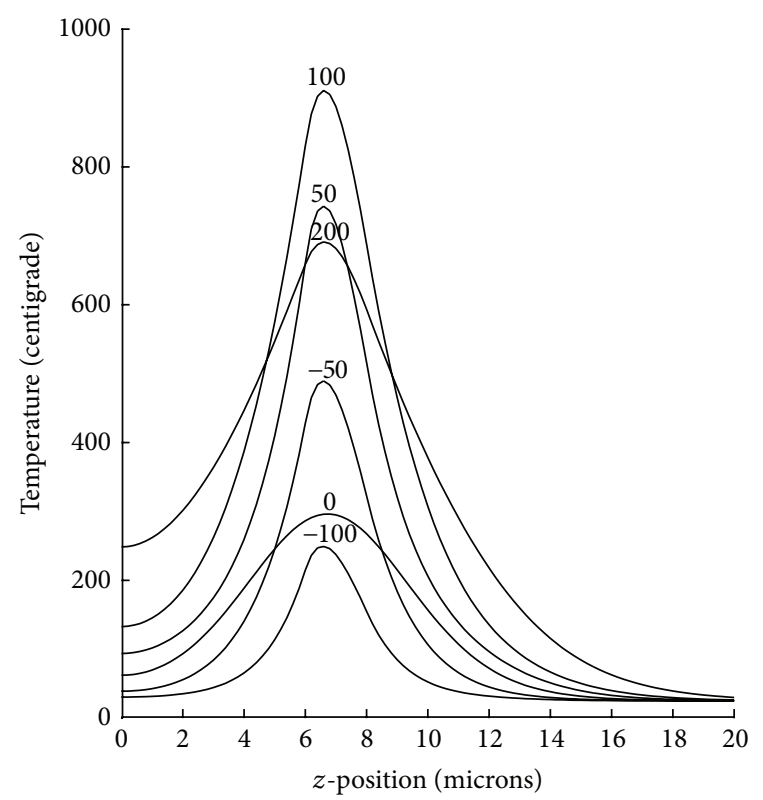

FIgURE 2: Temperature profiles on $x=2.5 \mu \mathrm{m}, y=0$ as a function of $z$ at times $-100,-50,0,50,100$, and $200 \mu$ s.

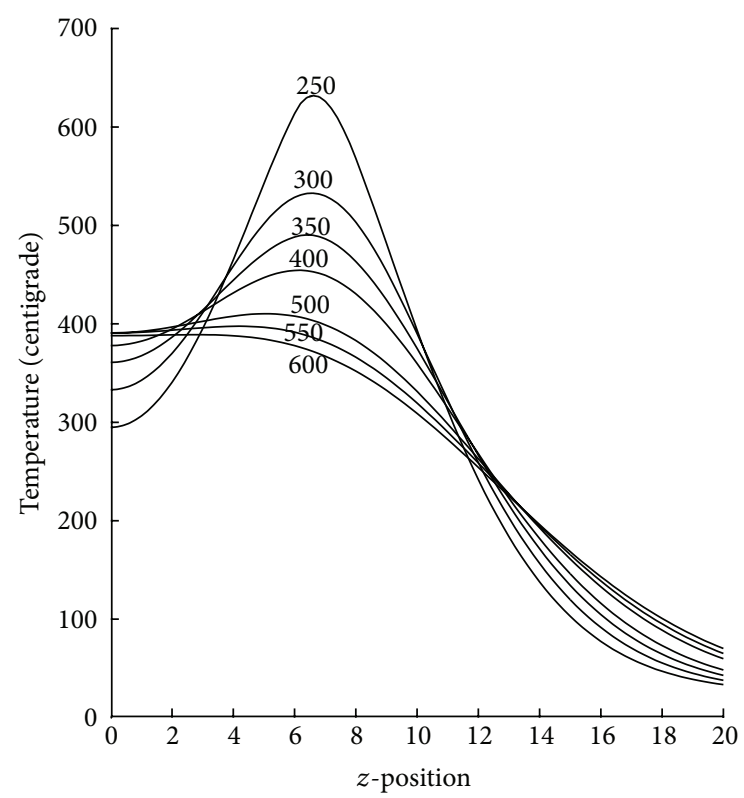

Figure 3: Temperature profiles on $x=2.5 \mu \mathrm{m}, y=0$ as a function of $z$ at times $250,300,350,400,500,550$, and $600 \mu \mathrm{s}$.

The laser is turned on at $\tau=0$ for $100 \mu \mathrm{s}$, but for much of that time, little heating occurs at $x=2.5 \mu \mathrm{m}$ since the laser beam has moved further to the right. Thus much lower temperatures of order $400^{\circ} \mathrm{C}$ are achieved at $t=50 \mu \mathrm{s}$. Figure 5 shows the typical cooling curves as heat conducts upwards and downwards from the dye coat layer. In Figure 8 the temperature profiles on $y=0, z=7$ micro meter and $v=0.1 \mathrm{~m} / \mathrm{s}$ as a function of $x$ for the different of time.

The diffusion coefficient will be much smaller at these lower temperatures, but there will be negligible interaction

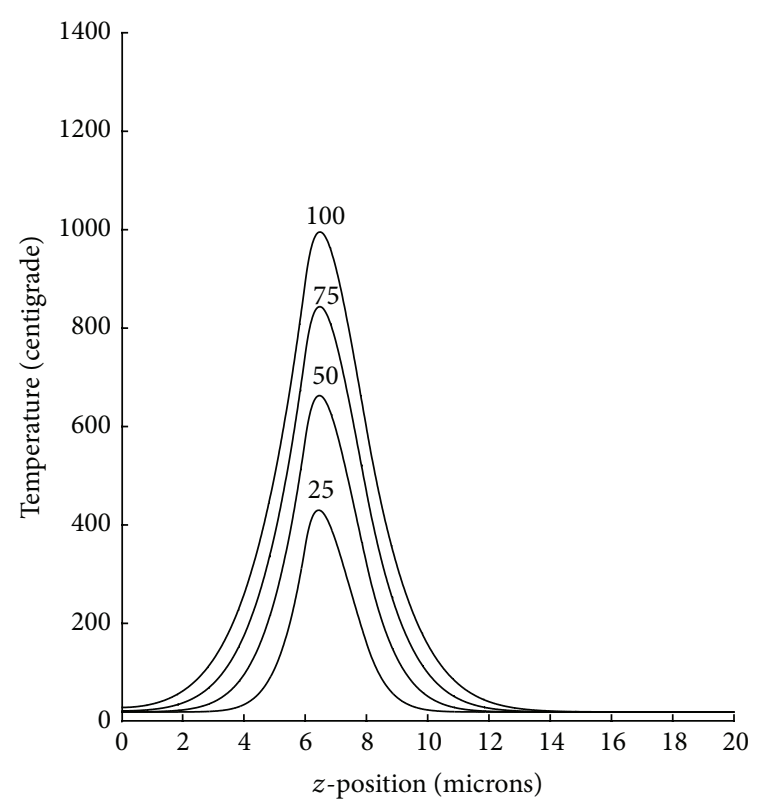

FIGURE 4: Temperature profiles on $r=0$ at times $t=25,50,75$, and $100 \mu$ s for an uniformly distributed absorber in the dye layer (heating phase). The laser is stationary.

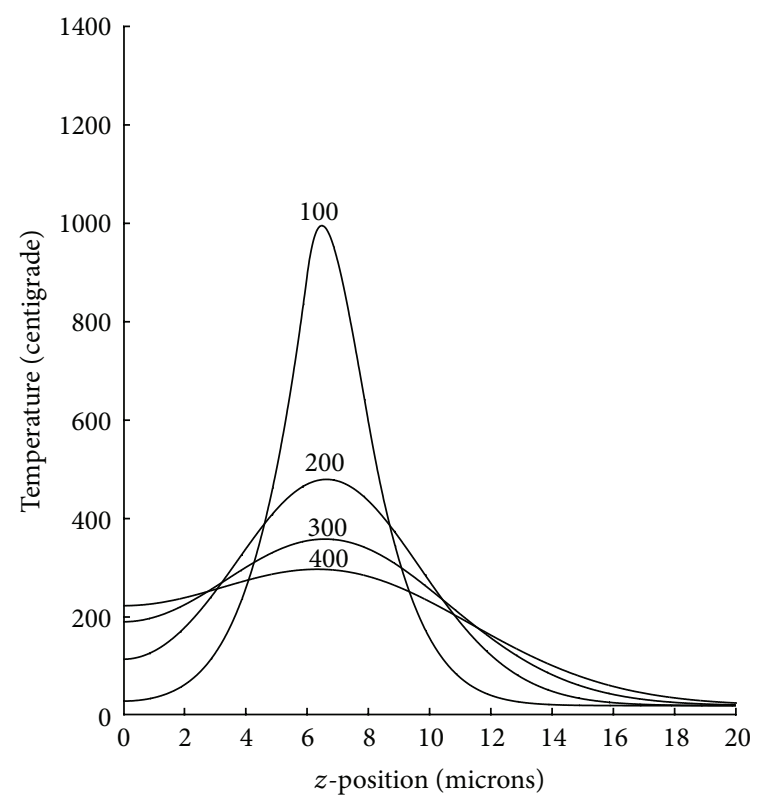

FIGURE 5: Temperature profiles on $r=0$ at times $t=100,200,300$, and $400 \mu$ s for an uniformly distributed absorber in the dye layer (cooling phase). The laser is stationary.

with other pulses. Higher temperatures could of course be achieved by increasing the laser wattage.

\section{Conclusions}

We have developed a simple theoretical model which allows us to calculate the $z$-temperature field in the dye coat layer produced by a moving laser beam. Our main conclusion is 


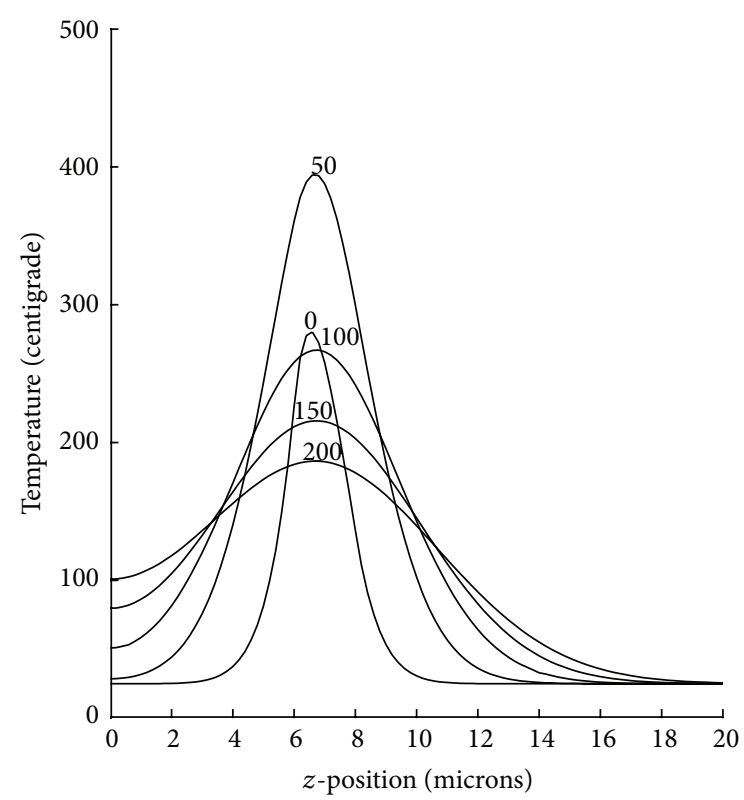

Figure 6: Temperature profiles on $x=2.5 \mu \mathrm{m}, y=0$, and $v=0.5$ as a function of $z$ at times $0,50,100,150$, and $200 \mu$ s.

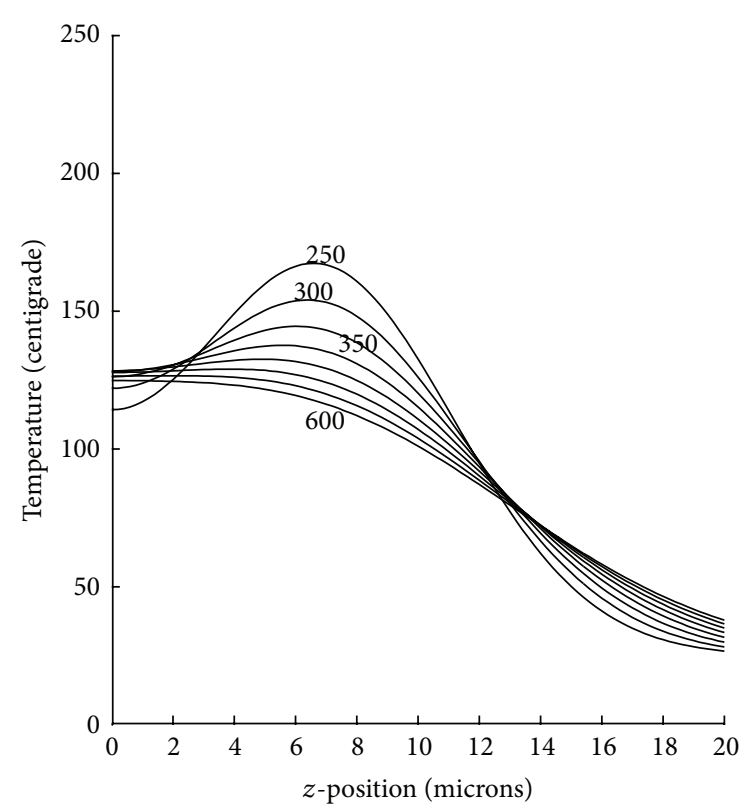

FIgURE 7: Temperature profiles on $x=2.5 \mu \mathrm{m}, y=0$, and $v=0.5$ as a function of $z$ at times $250,300,350,400,450,500,550$, and $600 \mu \mathrm{s}$.

that, for the standard data, the temperature at a given point is significantly influenced by at least two pulses leading to a complicated $z$-temperature profile in the dye coat layer as time changes. This implies that the dye-diffusion process is much more complicated than that predicted by a stationary laser model.

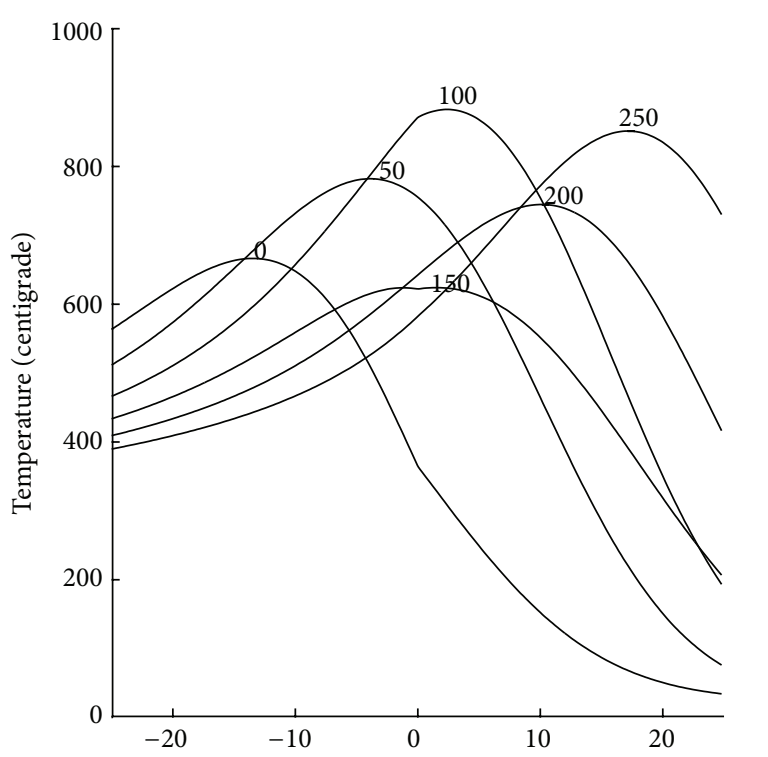

$x$-position (microns) ( $t$ start times $0.50,100,150,200$, and 250 )

Figure 8: Temperature profiles on $z=7, y=0$, and $v=0.1$ as a function of $x$ at times $0,50,100,150,200$, and $250 \mu$ s.

\section{Nomenclature}

$C_{p}:$ Specific heat

$h_{1}$ : Thickness of top melinex layer

$h_{2}$ : Thickness of dye-coat layer

$r_{0}$ : Radial length scale of laser beam

$\alpha:$ A typical thermal diffusivity

$t:$ Time

$t^{\prime}$ : Time at which heat source generated

$G$ : Instantaneous heat source solution

$T:$ Temperature

$\rho:$ Density

$T_{A}$ : Initial temperature

$\tau: \quad$ Time $\left(t-t^{\prime}\right)$

$v:$ Speed of laser beam

$W_{0}$ : Wattage of laser

$\mu$ : Optical density

$\lambda: \mu \ln 10$.

\section{Acknowledgments}

The author wishes to thank Dr. Richard Hann and Dr. Ken Hutt of ICI Imagedata for introducing them to this problem and for providing representative data.

\section{References}

[1] R. A. Hann and N. C. Beck, "Dye diffusion thermal transfer (D2T2) color printing," Journal of Imaging Technology, vol. 16, pp. 238-241, 1990.

[2] R. Uyhan and J. A. King-Hele, "Dye diffusion by laser heating," Mathematical Engineering in Industry, vol. 8, no. 4, pp. 275-292, 2002. 
[3] W. Hutt, I. R. Stephenson, H. C. V. Tran, A. Kaneko, and R. A. Hann, "Laser dye transfer," in Proceedings of the IS \& $T$ 8th International Congress on Advances in Non-Impact Printing Technologies, pp. 367-369, 1992.

[4] N. C. Beck and R. A. Hann, "Thermal transfer printing project: measurement of the diffusion coefficients of dyes in a range of polyesters," ICI Imagedata report, 1986.

[5] G. Araya and G. Gutierrez, "Analytical solution for a transient, three-dimensional temperature distribution due to a moving laser beam," International Journal of Heat and Mass Transfer, vol. 49, no. 21-22, pp. 4124-4131, 2006.

[6] P. J. Cheng and S. C. Lin, "Analytical model for the temperature field in the laser forming of sheet metal," Journal of Materials Processing Technology, vol. 101, no. 1, pp. 260-267, 2000.

[7] R. Brockmann, K. Dickmann, P. Geshev, and K.-J. Matthes, "Calculation of temperature field in a thin moving sheet heated with laser beam," International Journal of Heat and Mass Transfer, vol. 46, no. 4, pp. 717-723, 2003.

[8] H. S. Carslaw and J. C. Jaeger, Conduction of Heat in Solids, Clarendon Press, Oxford, UK, 1946.

[9] R. Uyhan and J. A. King-Hele, "Modelling of thermal printers," Applied Mathematical Modelling, vol. 32, no. 4, pp. 405-416, 2008. 


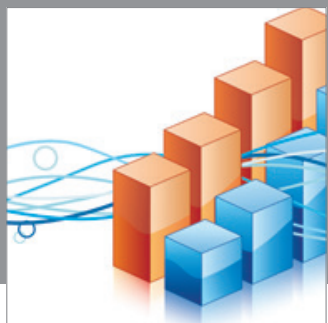

Advances in

Operations Research

mansans

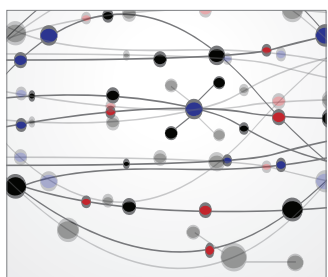

The Scientific World Journal
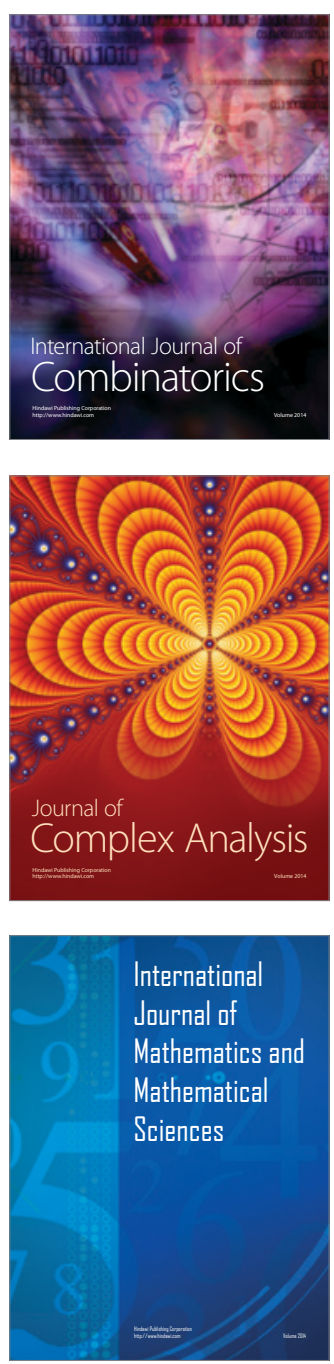
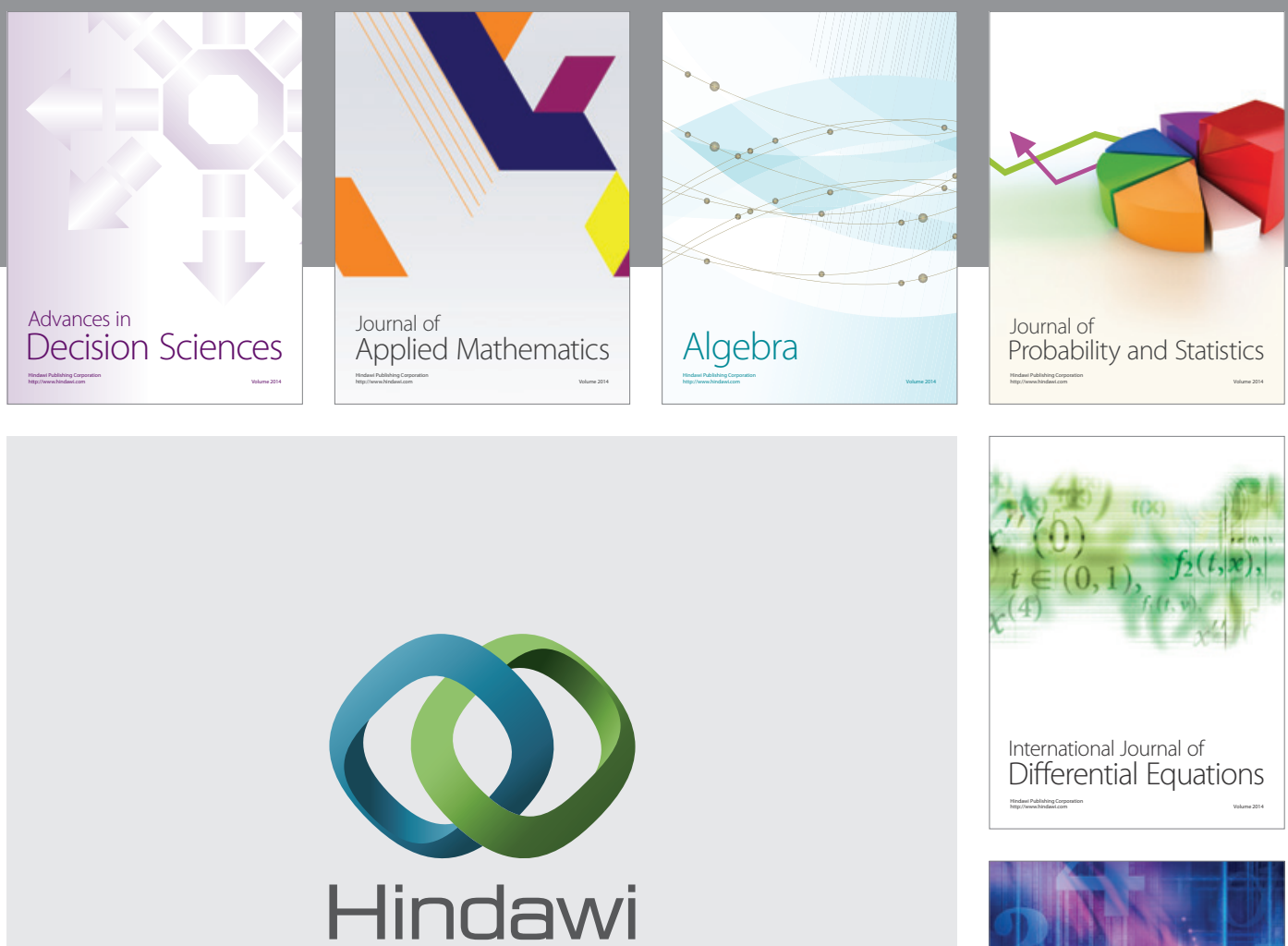

Submit your manuscripts at http://www.hindawi.com
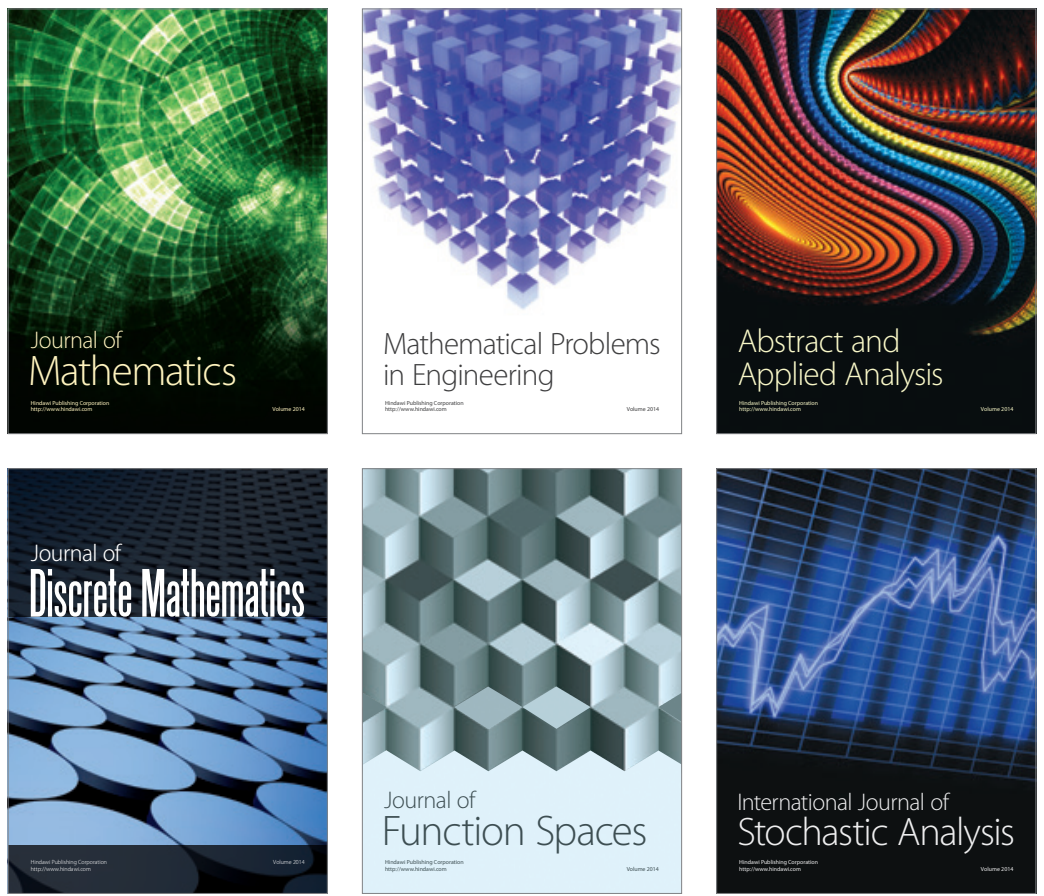

Journal of

Function Spaces

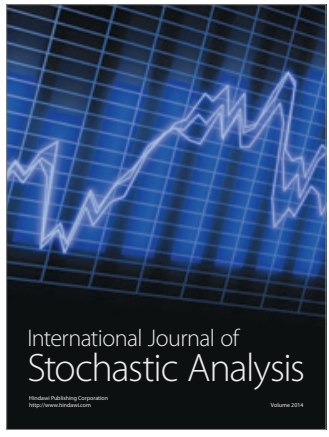

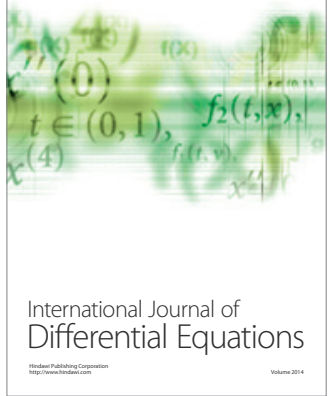
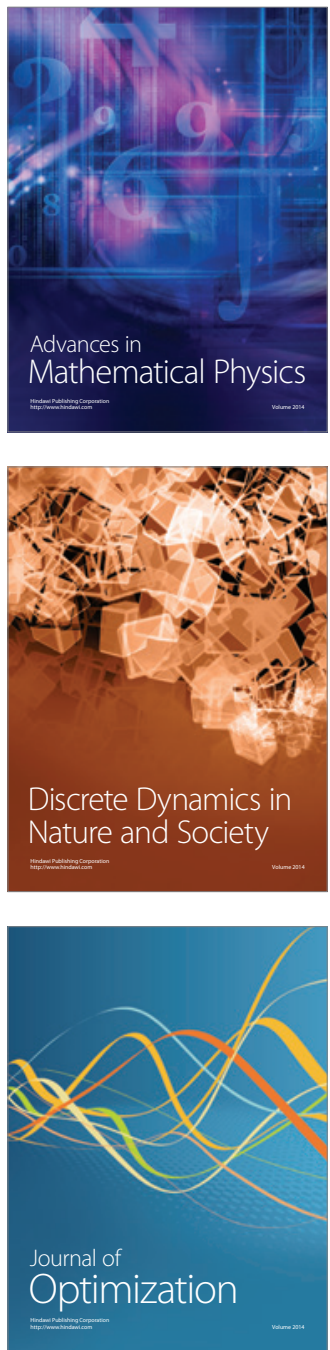